\title{
Novel oncolytic adenovirus sensitizes renal cell carcinoma cells to radiotherapy via mitochondrial apoptotic cell death
}

\author{
REN-FU CHEN ${ }^{1 *}$, YUE-YAN LI $^{1 *}$, LIAN-TAO LI $^{2}$, QIAN CHENG ${ }^{2}$, GUAN JIANG ${ }^{2}$ and JUN-NIAN ZHENG ${ }^{2}$ \\ ${ }^{1}$ Department of Urology, Affiliated Hospital of Xuzhou Medical College; \\ ${ }^{2}$ Jiangsu Key Laboratory of Biological Cancer Therapy, Xuzhou Medical College, Xuzhou, Jiangsu 221002, P.R. China
}

Received November 18, 2013; Accepted July 4, 2014

DOI: $10.3892 / \mathrm{mmr} .2014 .2987$

\begin{abstract}
Renal cell carcinoma is the most frequent kidney malignancy and patients with metastatic disease have a poor prognosis. Suppressed apoptosis and marked invasiveness are distinctive features of renal cell carcinoma. In the present study, a dual-regulated oncolytic adenovirus expressing the interluekin (IL)-24 gene (Ki67-ZD55-IL-24) was constructed utilizing the $\mathrm{Ki} 67$ promoter to replace the native viral promoter of the E1A gene. Whether the combination of Ki67-ZD55-IL-24-mediated gene virotherapy and radiotherapy produced increased cytotoxicity in renal cell carcinoma cells via mitochondrial apoptotic cell death was investigated. The data indicated that this novel strategy has the potential to be further developed into an effective approach to treat renal cell carcinoma. The results showed that the combination of Ki67-ZD55-IL-24 and radiotherapy significantly enhanced anti-tumour activity via increasing the induction of apoptosis in melanoma cells compared with the other agents.
\end{abstract}

\section{Introduction}

Renal cell carcinoma (RCC) is the most frequent kidney malignancy and patients with metastatic RCC have a poor prognosis (1). In the United States, RCC is diagnosed in $\sim 51,000$ patients each year (2). If the disease is detected at an early stage, a large portion of the kidney or the entire organ may be removed, and prolonged patient survival may be achieved (3). However, if the disease has spread beyond the capsule of the kidney into the adrenal gland or surrounding

Correspondence to: Dr Jun-Nian Zheng or Dr Guan Jiang, Jiangsu Key Laboratory of Biological Cancer Therapy, Xuzhou Medical College, 84 West Huai-Hai Road, Xuzhou, Jiangsu 221002, P.R. China

E-mail: jnzheng@xzmc.edu.cn

*Contributed equally

Key words: renal cell carcinoma, oncolytic adenoviruses, interleukin-24, radiation, apoptosis fascia with nodal involvement, the prognosis is poor with rapid progression. In addition, RCC is frequently characterized as highly refractory to multiple established cytotoxic radiotherapy and chemotherapy regimens.

Previous data have suggested that interleukin 24 (IL-24) is a promising candidate for cancer gene therapy (4). IL-24 has been demonstrated to suppress growth and induce apoptosis in a wide range of human cancer types without apparent cytotoxicity to normal cells (4). Recent studies have shown that IL-24 radiosensitizes various cancer cells, including non-small cell lung carcinoma, renal carcinoma and malignant glioma cells (5).

$\mathrm{Ki}-67$ is an established proliferation mediator and is used extensively to estimate the proliferation fraction of tumors (6). The Ki67 labeling index is an independent predictor of disease progression and recurrence in carcinomas, and also is used as a grade index and prognostic marker. The $\mathrm{Ki}-67$ gene promoter is a tumor-selective promoter with the desired specificity and efficiency to further restrict transgene expression in tumor cells and improve the targeting of gene therapy (7).

Oncolytic adenoviruses are a novel class of therapeutic agent in cancer treatment. A previous study observed that the E1B 55-kDa gene-defective oncolytic adenovirus ZD55 not only efficiently infected, replicated in and lysed tumor cells, but also amplified IL-24 gene expression levels. Furthermore, the increased expression levels of IL-24 in the tumor microenvironment did not affect adjacent normal cells (8).

One strategy to achieve the desired tumor selectivity is the employment of tumor-specific transcriptional response elements (promoters) to regulate the expression levels of early viral genes that are critical for replication, such as E1A. These are required to transactivate other adenoviral genes (9). Promising tumor-selective promoters may restrict the sites of viral replication and the expression of therapeutic transgenes. Therefore, the selection of specific promoters with tight regulation is important.

These recent studies prompted the development of a hypothesis speculating that a combination of a Ki67 promoter-controlled oncolytic adenovirus (Ki67-ZD55-IL-24) and radiation therapy may produce an enhanced antitumor effect against RCC cells compared with the effect of either agent used alone, which was investigated in the present study. 


\section{Materials and methods}

Cell lines and vectors. The Ketr-3 and 786-O renal cancer cell lines were purchased from the Shanghai Cell Collection (Shanghai, China). The cells were cultured in Dulbecco's modified Eagle's medium (Gibco-BRL, Grand Island, NY, USA) supplemented with $10 \%$ heat-inactivated fetal bovine serum (Gibco-BRL), $4 \mathrm{mM}$ glutamine, $50 \mathrm{U} / \mathrm{ml}$ penicillin and $50 \mu \mathrm{g} / \mathrm{ml}$ streptomycin at $37^{\circ} \mathrm{C}$ in a humidified atmosphere with $5 \% \mathrm{CO}_{2}$. The cells were screened routinely to verify the lack of mycoplasma contamination for use in the long phase of growth.

The recombinant adenoviruses used in the present study, including Ki67 promoter-controlled ZD55 carrying IL-24 (Ki67-ZD55-IL-24), Ki67 promoter-controlled ZD55 (Ki67-ZD55) and ZD55 carrying IL-24 (ZD55-IL-24), have been previously described $(8,10)$. The Ki67 promoter used in the present study was previously constructed (11). The viral plaques were observed 9-12 days after transient transfection. These recombinant adenoviruses were verified by reverse transcription-polymerase chain reaction (RT-PCR). Viruses were plaque-purified and propagated in HEK293 cells, and functional plaque-forming unit (PFU) titers were determined by a plaque assay using an Adeno-XTM Rapid Titer kit (Clontech Laboratories, Palo Alto, CA, USA) in HEK293 cells. The PFU titers of the adenoviruses were found to be $3.5 \times 10^{10} \mathrm{PFU} / \mathrm{ml}$.

Radiation. An X-irradiator (Model X.S.S.205 FZ; Hangzhou Jiancheng Trade Co. Ltd., Hangzhou, China) was used in the present study. The dose rate was $0.287 \mathrm{~Gy} / \mathrm{min}$ with $200 \mathrm{kV} / 10 \mathrm{~mA}$ using 0.5-mm-thick Cu/0.5-mm-thick Al filters, and the distance between the X-ray source and target was $56 \mathrm{~cm}$. The renal cancer cells were irradiated with 0.5-6 Gy ionizing radiation using a $137 \mathrm{Cs}$ g-irradiation source $48 \mathrm{~h}$ after viral treatment.

Immunocytochemical staining. Ketr-3 and 786-O cells were fixed with $4 \%$ paraformaldehyde onto glass coverslips. Subsequent to washing with phosphate-buffered saline (PBS), the cells were incubated with anti-Ki67 monocloncal mouse antibody (Santa Cruz Biotechnology, Santa Cruz, CA, USA) for $24 \mathrm{~h}$ and then incubated with a horseradish peroxidase-conjugated goat anti-rabbit monoclonal secondary antibody (ZSGB-BIO, Beijing, China) for $1 \mathrm{~h}$ followed by colorimetric detection using diaminobezidine (Sigma-Aldrich, St. Louis. MO, USA). For evaluation of the Ki67-positive fraction, at least 200 cells were counted using a microscope (CH-2 CHT; Olympus, Tokyo, Japan) in six regions and the mean number was determined.

Cell viability assay. Ketr-3 and 786-O cells were plated at a density of $10^{5}$ cells $/ 6 \mathrm{~cm}$ dish and treated with radiation alone (5 Gy or $10 \mathrm{~Gy}$ ), ZD55-IL-24 alone [0, 0.1, 1, 10 or 100 multiplicity of infection (MOI)], ZD55-IL-24 plus radiation, Ki67-ZD55-IL-24 alone (0, 0.1, 1, 10 or $100 \mathrm{MOI})$ or Ki67-ZD55-IL-24 plus radiation at the indicated dosages. Subsequent to treatment for four days with the indicated doses, cell survival rates were evaluated by standard MTT assay (Sigma-Aldrich) according the manufacturer's instructions.
Four replicate wells were examined per assay and each experiment was repeated three times.

Apoptotic cell staining. Ketr-3 and 786-O cells were seeded in flat-bottomed 6-well plates and were treated with Ki67-ZD55-IL-24, ZD55-IL-24 or the two agents combined. Untreated cells served as controls. After $48 \mathrm{~h}$ treatment, the cells were incubated with Hoechst 33258 (Nanjing Keygen Biotech, Nanjing, China) for $10 \mathrm{~min}$, washed with PBS twice and observed under a fluorescence microscope (Nikon, Midori, Japan).

Evaluation of apoptosis. Apoptosis was determined by staining cells with Annexin V-fluorescein isothiocyanate (FITC) and propidium iodide (PI) using an Annexin V-FITC apoptosis detection kit (BD Pharmingen, San Diego, CA, USA). The cells were treated with Ki67-ZD55-IL-24, ZD55-IL-24 or the two agents combined for $96 \mathrm{~h}$, and then harvested and washed twice with cold PBS. The prepared cells were resuspended in binding buffer (containing $10 \mathrm{mM} \mathrm{HEPES/NaOH}$ ( $\mathrm{pH} 7.4$ ), $140 \mathrm{mM} \mathrm{NaCl}$ and $2.5 \mathrm{mM} \mathrm{CaCl}$ ) at a concentration of $1 \times 10^{6}$ cells per ml. Subsequently, $5 \mathrm{ml}$ Annexin V-FITC (BD Pharmingen) and $5 \mathrm{ml}$ PI were added to these cells, which were analyzed by FACStar flow cytometer (Becton Dickinson, Franklin Lakes, NJ, USA).

Western blot analysis. The cells were harvested from the plates and aliquots of cell extracts were separated on a $12 \%$ SDS-polyacrylamide gel. The proteins were then transferred to a nitrocellulose membrane and incubated overnight at $4^{\circ} \mathrm{C}$ with the following rabbit polyclonal antibodies: Anti-IL-24 (Gen Hunter C-corporation, Nashville, TN, USA), anti-caspase-3, anti-caspase-8, anti-Bcl-2, anti-Bax, anti-Mcl-1 (Cell Signaling Technology, Inc., Beverly, MA, USA), anti- $\beta$-actin and anti-E1A (SantaCruzBiotechnology).Themembraneswerethenwashedand incubated with alkaline phosphatase-conjugated goat anti-rabbit monoclonal secondary antibodies (ZSGB-BIO) in Tris-buffered saline and Tween-20 for $2 \mathrm{~h}$ and developed using nitro blue tetr azolium/5-bromo-4-chloro-3-indolyl-phosphate color substrate (Promega Corporation, Madison, WI, USA). The bands on the membrane were scanned and their density was analyzed with an Image-J analyzer (LabWorks Software; UVP, Upland, CA, USA).

Statistical analysis. Data are expressed as the mean \pm standard deviation, and were analyzed by statistical software (SPSS Base 17.0 for Windows; SPSS, Inc., Chicago, IL, USA) using independent sample Student's t-test and analysis of variance, as appropriate. $\mathrm{P}<0.05$ was considered to indicate a statistically significant difference.

\section{Results}

Ki67 protein is overexpressed in tumor sections, as detected by immunohistochemical staining. The expression levels of Ki67 protein in renal cancer cells were examined. Ketr-3 and 786-O cells were frozen and subjected to immunohistochemical analysis of Ki67 expression. Compared with the HK-2 normal renal tube cells, Ketr-3 and 786-O cells exhibited significant overexpression of Ki67 (P<0.05; Fig. 1). 


\section{Ki67}

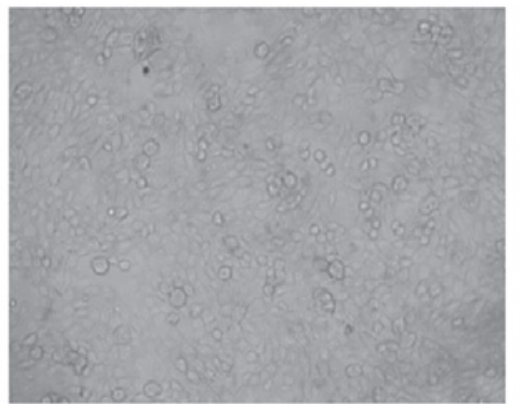

HK-2

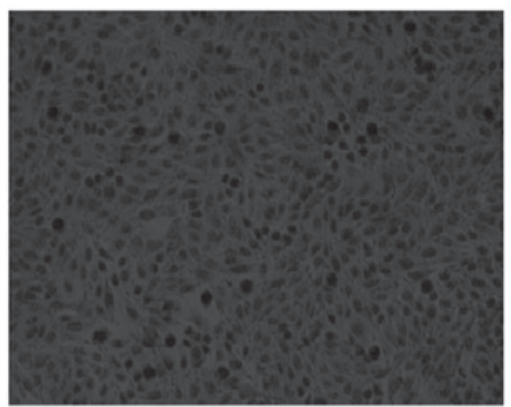

Ketr-3

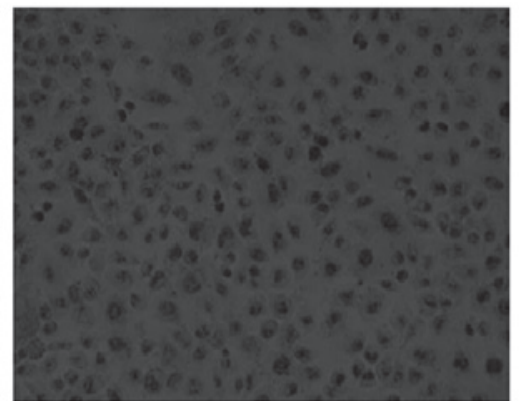

$786-\mathrm{O}$

Figure 1. Expression of Ki67 in the Ketr-3 and 786-0 renal cancer cell lines, along with the HK-2 normal renal tube cell line, was assessed by immunohistochemical analysis. Ki67 protein levels were analyzed using the anti-Ki67 antibody (original magnification, x400).
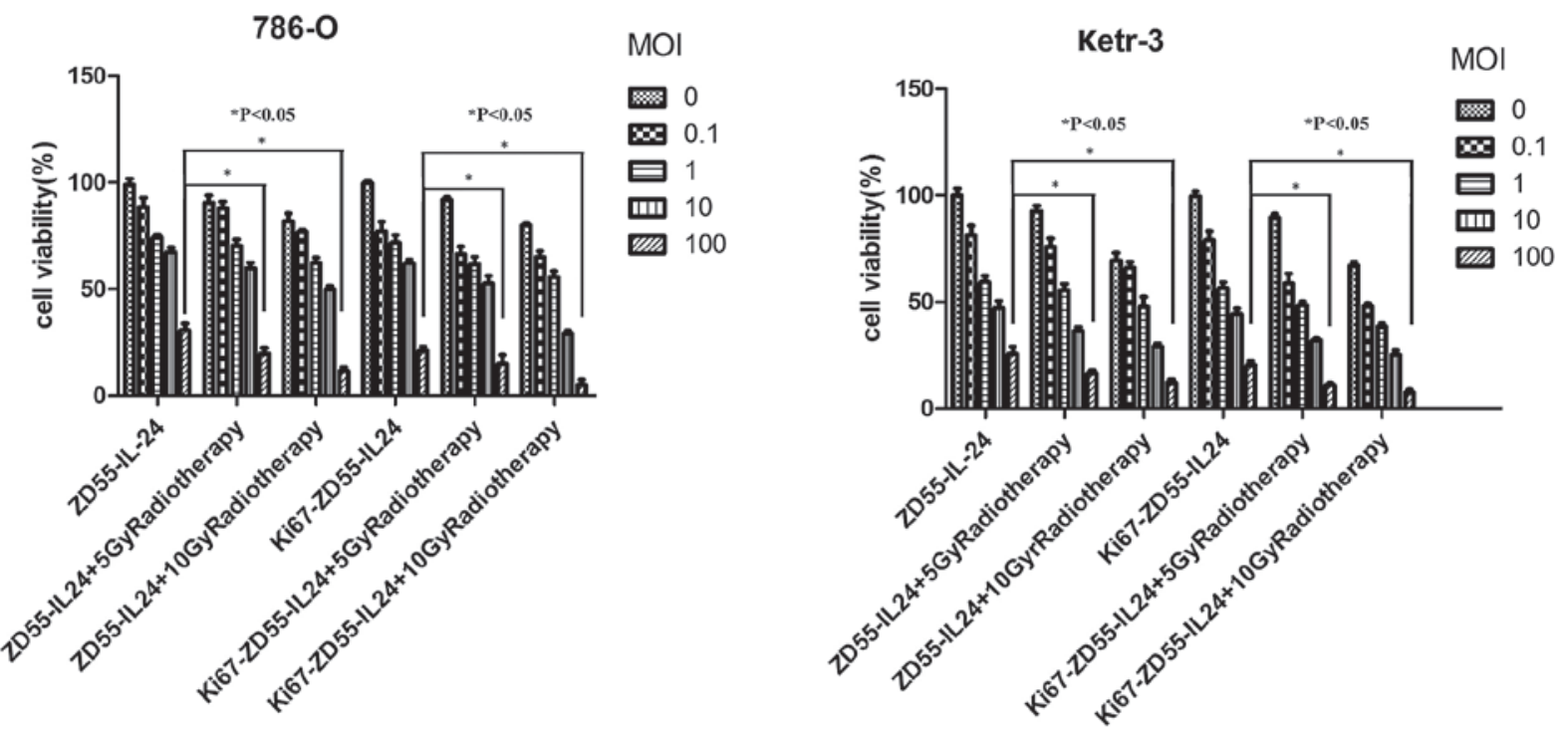

Figure 2. Combination Ki67-ZD55-interleukin (IL)-24 and radiotherapy treatment inhibited the growth of 786-O and Ketr-3 renal cancer cells. The cells were treated with Ki67-ZD55-IL-24, Ki67-ZD55-IL-24 plus radiotherapy, ZD55-IL-24 or ZD55-IL-24 plus radiotherapy. The radiotherapy dose was 5 or 10 Gy. The viruses were administered at the indicated multiplicity of infection (MOI) dosages. The cells were subjected to MTT assay on day four after treatment. Results are expressed as the mean \pm standard deviation (error bars) by the percentage of untreated control cells. "P<0.05 vs. Ki67-ZD55-IL-24 and ZD55-IL-24-treated groups ( $\mathrm{n}=6)$.

\section{$786-\mathrm{O}$}
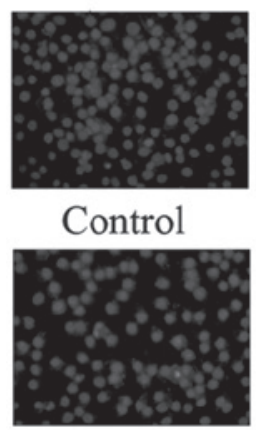

Radiotherapy

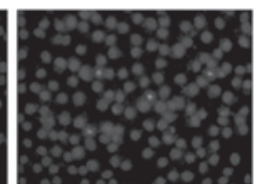

ZD55-IL24

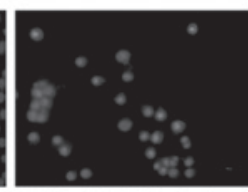

ZD55-IL24+ Radiotherapy

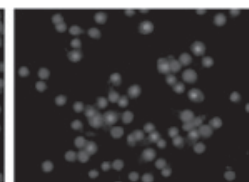

Ki67-ZD55-IL24

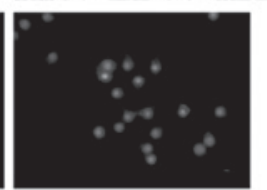

Ki67-ZD55-IL24+ Radiotherapy
Ketr-3
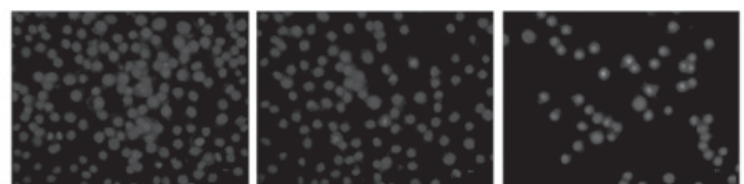

Control

ZD55-IL24 Ki67-ZD55-IL24
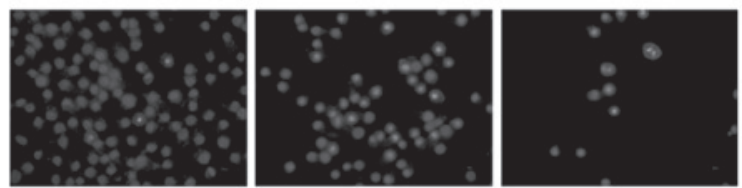

Radiotherapy
ZD55-IL24+ Ki67-ZD55-IL24+ Radiotherapy Radiotherapy

Figure 3. Morphological changes in the nuclei examined by Hoechst 33258 staining. 786-O and Ketr-3 renal cancer cells were treated with Ki67-ZD55-interleukin (IL)-24, Ki67-ZD55-IL-24 plus radiation, ZD55-IL-24, ZD55-IL-24 plus radiation or radiation alone. After $48 \mathrm{~h}$, the cells were incubated with Hoechst 33258 for $2 \mathrm{~h}$, and nuclei condensation and fragmentation were observed under a fluorescence microscope (original magnification, $\mathrm{x} 400 ;$ scale bar, $20 \mu \mathrm{m}$ ). 

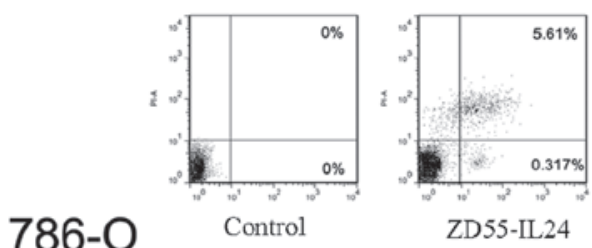

ZD55-IL24

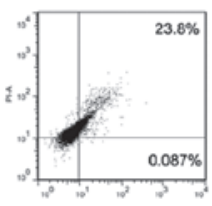

Radiotherapy

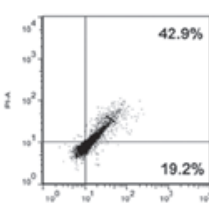

ZD55-IL24+

Radiotherapy

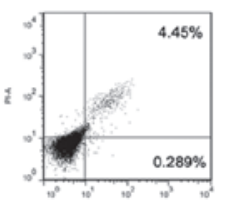

Ki67-ZD55-IL24

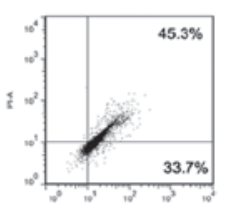

Ki67-ZD55-1L24+ Radiotherapy
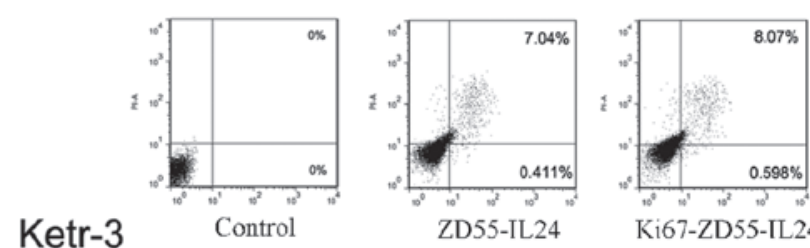

ZD55-IL24
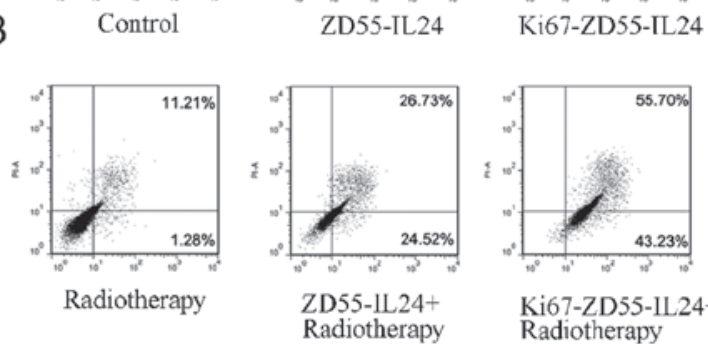

Radiotherapy

ZD55-IL24+
Radiotherapy

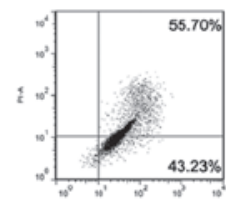

Ki67-ZD55-IL24+ Radiotherapy

Figure 4. Induced apoptotic changes examined by Annexin V-fluorescein isothiocyanate (FITC) staining. 786-O and Ketr-3 renal cancer cells were treated with Ki67-ZD55-interleukin (IL)-24, Ki67-ZD55-IL-24 plus radiation, ZD55-IL-24, ZD55-IL-24 plus radiation or radiation alone. Phosphate-buffered saline treatment served as a negative control. After 96 h, cells were stained with FITC-labeled Annexin V and immediately analyzed by flow cytometry.

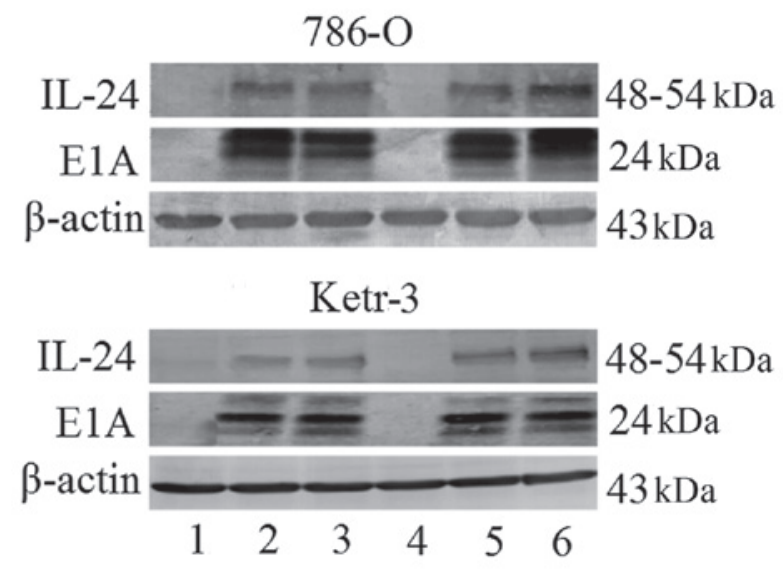

Figure 5. Ki67 promoter-controlled oncolytic adenoviruses induced efficient delivery of interleukin (IL)-24 in 786-O and Ketr-3 renal cancer cells. Cells were treated with Ki67-ZD55-IL-24, Ki67-ZD55-IL-24 plus radiation, ZD55-IL-24, ZD55-IL-24 plus radiation or radiation alone; phosphate-buffered saline (PBS) treatment served as a negative control. After $72 \mathrm{~h}$ treatment, IL-24 and E1A protein expression levels were analyzed by western blotting using antiIL-24 and E1A antibodies. $\beta$-actin served as a loading control. Lane 1, PBS; lane 2, ZD55-IL-24; lane 3, Ki67-ZD55-IL-24; lane 4, radiotherapy; lane , ZD55-IL-24 + radiotherapy; and lane 6, Ki67-ZD55-IL-24 + radiotherapy.

Ki67-ZD55-IL-24 combined with radiotherapy results in loss of cell viability. To investigate whether Ki67-ZD55-IL-24 in combination with radiotherapy inhibits the proliferation of renal cancer cells, the cells were plated in 96-well plates and treated with 5 or 10 Gy radiotherapy alone, ZD55-IL-24 alone, Ki67-ZD55-IL-24 (0, 0.1, 1, 10 or 100 MOI) alone, ZD55-IL-24 plus radiation or Ki67-ZD55-IL-24 plus radiation. Four days following treatment, cell viability was determined by an MTT assay. As shown in Fig. 2, the combinatorial treatments resulted in enhanced inhibition of Ketr-3 and 786-O cell proliferation in a dose-dependent manner. In addition, for each concentration examined, treatment with Ki67-ZD55-IL-24 plus radiotherapy resulted in significantly greater inhibition than that of either Ki67-ZD55-IL-24 or ZD55-IL-24 treatment alone $(\mathrm{P}<0.05)$.

Differences in cell apoptotic staining detected following ZD55-IL-24 and radiotherapy treatment in renal cancer

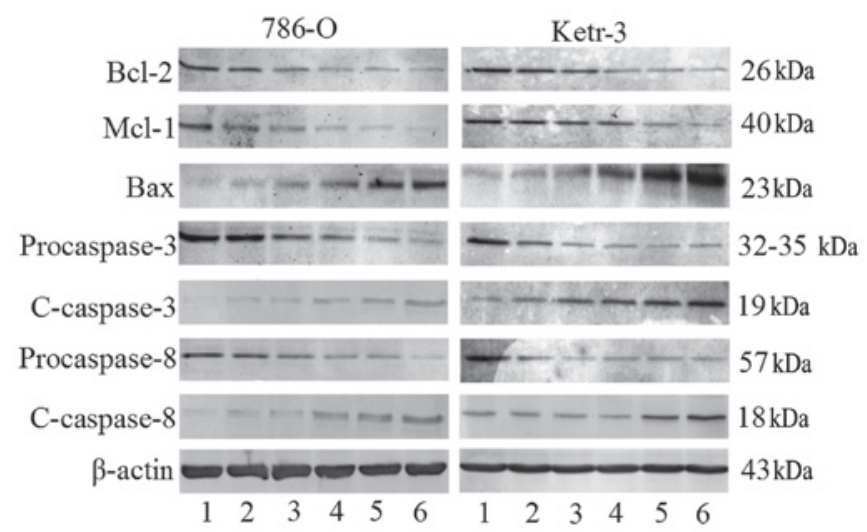

Figure 6. Ki67-ZD55-interleukin (IL)-24 sensitizes 786-O and Ketr-3 renal cancer cells to radiotherapy via mitochondrial apoptotic cell death. Renal cancer cells were treated with Ki67-ZD55-IL-24, Ki67-ZD55-IL-24 plus radiation, ZD55-IL-24 plus radiation or radiation alone; phosphate-buffered saline (PBS) treatment served as a negative control. After $72 \mathrm{~h}$ treatment, cell extracts were subjected to western blot analysis of caspase- 8 and caspase-3 expression levels. The anti-apoptotic proteins Bcl-2 and Mcl-1, and the pro-apoptotic protein Bax expression levels were also examined. $\beta$-actin served as a loading controls $(n=6)$. Lane 1, PBS; lane 2, ZD55-IL-24; lane 3, Ki67-ZD55-IL24; lane 4, radiotherapy; lane 5, ZD55-IL-24 + radiotherapy; and lane 6, Ki67-ZD55-IL24 + radiotherapy.

cells. Ketr-3 and 786-O cells treated with radiation alone, virus alone, and the combination of radiation and the virus were observed, using Hoechst 33258 staining, to be markedly different in cellular and nuclear morphology, such as DNA fragmentation and chromatin condensation, compared with control (Fig. 3).

Ki67-ZD55-IL-24 and radiotherapy contribute to apoptosis in renal cancer cells. To analyze whether the enhanced cytotoxicity resulting from Ki67-ZD55-IL-24 and radiotherapy treatment was due to increased apoptosis, the apoptotic changes in Ketr-3 and 786-O cells were examined following different treatment schemes at an MOI of 10 for $96 \mathrm{~h}$. Enhanced permeability of the cellular membrane, an early sign of apoptosis, was assessed by flow cytometric analysis using Annexin V-FITC staining. After $96 \mathrm{~h}$ treatment, the 
radiotherapy alone and ZD55-IL-24, Ki67-ZD55-IL-24 and ZD55-IL-24 plus radiotherapy treatments caused 12.49, 7.45, 8.67 and $51.25 \%$ Ketr-3 cells respectively to lose membrane integrity. The Ki67-ZD55-IL-24 in combination with radiotherapy treatment resulted in $98.93 \%$ of Ketr-3 cells to lose membrane integrity, which was significant $(\mathrm{P}<0.05$; Fig. 4). A similar result was observed in 786-O cells.

Ki67 promoter-controlled oncolytic adenoviruses induce efficient delivery of IL-24 in renal cancer cells. IL-24 protein levels were determined by western blotting. After $48 \mathrm{~h}$ treatment, IL-24 was stably overexpressed in 786-O and Ketr-3 cells and no discernible difference in IL-24 expression levels was observed in cells treated with either Ki67-ZD55-IL-24 alone or Ki67-ZD55-IL-24 plus radiotherapy (Fig. 5), demonstrating that Ki67-ZD55 mediated a high and stable expression level of IL-24, and that radiotherapy did not affect IL-24 expression levels. Furthermore, similar expression levels of the adenoviral E1A protein were detected in Ki67-ZD55-IL-24-infected cells with or without radiotherapy (Fig. 5), indicating that Ki67 promoter-controlled oncolytic adenoviruses replicated productively in renal cancer cells and that radiotherapy did not attenuate the replicative ability of the virus.

Ki67-ZD55-IL-24 sensitizes renal cancer cells to radiotherapy via mitochondrial apoptotic cell death. To elucidate the mechanism underlying the apoptosis observed following combination treatment, the levels of proteins in the mitochondrial apoptotic pathway were monitored by western blotting following treatment of Ketr-3 and 786-O cells with radiation alone, virus alone, or a combination of radiation and the virus. Bcl-2 and Mcl-1 levels were observed to be decreased following Ki67-ZD55-IL-24 treatment alone, but were further reduced when Ki67-ZD55-IL-24 was administered in combination with radiotherapy (Fig. 6). In addition, the administration of either PBS or ZD55-IL-24 alone did not significantly increase Bax expression levels, whereas Ki67-ZD55-IL-24 or Ki67-ZD55-IL-24 plus radiotherapy did. Accordingly, pro-caspase-3 and pro-caspase- 8 were significantly reduced in the combined treatment groups (Fig. 6).

\section{Discussion}

In recent years, therapeutic regimens combining radiation with oncolytic adenoviruses have demonstrated great potential in cancer treatment. An E1B 55 kDa-deleted conditionally replicated adenovirus, ONYX-015, has been observed to potentiate radiation therapy, resulting in several pre-clinical studies analyzing the value of combining the two treatments (12). Several specific types of oncolytic adenovirus administered in combination with radiotherapy have been observed to exhibit greater antitumor effects than treatment with either therapy alone (13-15).

Oncolytic adenoviruses offer certain advantages over conventional cancer therapy and are a promising novel approach for human cancer treatment. One strategy to achieve the desired tumor selectivity is the use of tumor-specific transcriptional response elements (promoters) to mediate the expression levels of early virus genes essential for replication, such as E1A, which are required to transactivate the other adenoviral genes (9).
Tumor-selective promoters regulate the expression levels of viral genes in tumor cells but not in normal cells, thus the adenovirus selectively replicates in the tumor cells and kills these cells (16).

Ki67 has been selected as a potential target in cancer gene therapy, as this promoter is present in the majority of malignant cells but only marginally detected in the majority of normal cells. Furthermore Ki67 expression is associated with cell proliferation. In addition, IL-24 is a good candidate for sensitizing tumor cells to radiotherapy without exacerbating toxicity, due to tumor-specific anti-angiogenic, pro-apoptotic and growth-inhibitory activities (17-19). A novel oncolytic adenovirus with E1A gene controlled by Ki67 promoter has been constructed, expressing the IL-24 gene (10). The Ki67 promoter is a potent controlling element of E1A. In the present study, the specific antitumor effects of Ki67 promoter controlled oncolytic adenoviruses expressing IL-24 were analyzed in human renal carcinoma cells.

Western blot analysis confirmed higher expression levels of E1A and IL-24 proteins in cells treated with either oncolytic adenoviruses or oncolytic adenoviruses plus radiotherapy. These results indicated that the oncolytic adenoviruses efficiently replicated in the renal carcinoma cells and that radiotherapy did not significantly affect IL-24 expression levels. Consistent with the finding that oncolytic adenoviruses have a selective ability to replicate in tumor cells, the MTT assay revealed that the administration of Ki67 promoter-controlled oncolytic adenoviruses plus radiotherapy specifically induced cytopathic effects in renal carcinoma cells.

To further elucidate the mechanism of Ki67-ZD55-IL-24 and radiotherapy-induced cell death, the effect of Ki67-ZD55-IL-24, with and without radiotherapy, on the levels of Bcl-2, Bax, caspase- 8 and caspase- 3 proteins was determined by western blotting in renal carcinoma cells. Ki67-ZD55-IL-24 administered in combination with radiotherapy markedly reduced Bcl-2 and MCL-1 levels, significantly increased Bax levels, and induced an evidently greater activation of caspase- 8 and caspase- 3 . These results suggest that treatment with Ki67-ZD55-IL-24 combined with radiotherapy induced changes in the levels and ratio of pro-apoptotic to anti-apoptotic proteins in renal carcinoma cells.

In conclusion, these results demonstrated that Ki67 promoter-controlled oncolytic adenovirus expressing IL-24, in combination with radiotherapy, may provide a novel and effective approach in the treatment of renal cell carcinoma.

\section{Acknowledgements}

This study was supported by grants from the National Natural Science Foundation of China (grant nos. 81372916, 81372460 and 81101702), the 'Six Talent Peaks' Project of Jiangsu Province (grant no. 2013-WSN-014), the Key University Science Research Project of Jiangsu Province (grant nos. 11KJA320002 and 12KJA320001), the Science and Technology Department of Jiangsu province (grant nos. BK20141142, BK2013348 and BK2011207) and Xuzhou Medical Young Talents Project.

\section{References}

1. Garcia JA and Rini BI: Recent progress in the management of advanced renal cell carcinoma. CA Cancer J Clin 57: 112-125, 2007. 
2. Park MA, Hamed HA, Mitchell C, et al: A serotype 5/3 adenovirus expressing MDA-7/IL-24 infects renal carcinoma cells and promotes toxicity of agents that increase ROS and ceramide levels. Mol Pharmacol 79: 360-380, 2011.

3. Sunela KL, Kataja MJ, Lehtinen ET, et al: Prognostic factors and long-term survival in renal cell cancer patients. Scand J Urol Nephrol 43: 454-460, 2009.

4. Jiang G, Zhang L, Xin Y, et al: Conditionally replicating adenoviruses carrying mda-7/IL-24 for cancer therapy. Acta Oncol 51: 285-292, 2012

5. Emdad L, Lebedeva IV, Su ZZ, et al: Historical perspective and recent insights into our understanding of the molecular and biochemical basis of the antitumor properties of mda-7/IL-24. Cancer Biol Ther 8: 391-400, 2009.

6. Colozza M, Sidoni A and Piccart-Gebhart M: Value of Ki67 in breast cancer: the debate is still open. Lancet Oncol 11: 414-415, 2010.

7. Pei DS, Qian GW, Tian H, et al: Analysis of human Ki-67 gene promoter and identification of the Sp1 binding sites for Ki-67 transcription. Tumour Biol 33: 257-266, 2012.

8. Zhao L, Gu J, Dong A, et al: Potent antitumor activity of oncolytic adenovirus expressing mda-7/IL-24 for colorectal cancer. Hum Gene Ther 16: 845-858, 2005.

9. Liu J, Fang L, Cheng Q, et al: Effects of G250 promoter controlled conditionally replicative adenovirus expressing Ki67-siRNA on renal cancer cell. Cancer Sci 103: 1880-1888, 2012.

10. Jiang G, Jiang AJ, Cheng Q, et al: A dual-regulated oncolytic adenovirus expressing interleukin-24 sensitizes melanoma cells to temozolomide via the induction of apoptosis. Tumour Biol 34 1263-1271, 2013.
11. Wang MJ, Pei DS, Qian GW, et al: p53 regulates Ki-67 promoter activity through p53- and Sp1-dependent manner in HeLa cells. Tumour Biol 32: 905-912, 2011

12. Geoerger B, Grill J, Opolon P, et al: Potentiation of radiation therapy by the oncolytic adenovirus dl1520 (ONYX-015) in human malignant glioma xenografts. Br J Cancer 89: 577-584, 2003.

13. Freytag SO, Barton KN, Brown SL, et al: Replication-competent adenovirus-mediated suicide gene therapy with radiation in a preclinical model of pancreatic cancer. Mol Ther 15: 1600-1606, 2007.

14. Nandi S, Ulasov IV, Tyler MA, et al: Low-dose radiation enhances survivin-mediated virotherapy against malignant glioma stem cells. Cancer Res 68: 5778-5784, 2008.

15. Kuroda S, Fujiwara T, Shirakawa Y, et al: Telomerase-dependent oncolytic adenovirus sensitizes human cancer cells to ionizing radiation via inhibition of DNA repair machinery. Cancer Res 70: 9339-9348, 2010.

16. Yu de B, Zhong SY, Yang M, et al: Potent antitumor activity of double-regulated oncolytic adenovirus-mediated ST13 for colorectal cancer. Cancer Sci 100: 678-683, 2009.

17. Bhutia SK, Das SK, Azab B, et al: Targeting breast cancer-initiating/stem cells with melanoma differentiation-associated gene-7/interleukin-24. Int J Cancer 133: 2726-2736, 2013.

18. Eulitt PJ, Park MA, Hossein H, et al: Enhancing mda-7/IL-24 therapy in renal carcinoma cells by inhibiting multiple protective signaling pathways using sorafenib and by Ad.5/3 gene delivery. Cancer Biol Ther 10: 1290-1305, 2010.

19. Park MA, Walker T, Martin AP, et al: MDA-7/IL-24-induced cell killing in malignant renal carcinoma cells occurs by a ceramide/CD95/PERK-dependent mechanism. Mol Cancer Ther 8: 1280-1291, 2009. 